\title{
An anisotropic mesh parameterization scheme
}

Received: 18 September 2002/ Accepted: 3 October 2003 / Published online: 20 May 2004

(C) Springer-Verlag London Limited 2004

\begin{abstract}
We introduce a simple anisotropic modification of Floater's shape-preserving parameterization scheme. The original scheme is formulated as a discrete energy minimization and the modification is performed by introducing an additional stretching term. Results and example applications to anisotropic regular surface meshing are presented.
\end{abstract}

Keywords Structured surface mesh generation • Anisotropy $\cdot$ Remeshing $\cdot$ Surface parameterization

\section{Introduction}

Surface meshes are widely used in manufacturing, medical and scientific applications. Acquired with shapeacquisition techniques, these meshes are often resampled into more regular representations $[1,2]$ to become more amenable for further usage. Creation of such regular representations is called remeshing, and it often involves patchwise parameterization of original mesh data onto simple planar regions. Recently, there has been a bout of interest in surface mesh parameterization algorithms targeting surface texturing $[3,4]$, geometry approximation with semiregular approximations [5,6], as well as general mesh parameterization techniques $[7,8]$.

In this paper we introduce a modification to a wellknown shape-preserving parameterization scheme of Floater [9]. We work in a setting useful to traditional remeshing algorithms that split the original surface mesh into topologically simple patches, and map each patch onto a simple planar region. We therefore restrict our attention to the case of a single mesh region mapped onto a square.

I. Guskov

University of Michigan,

Ann Arbor, MI 48109, USA

E-mail:guskov@eecs.umich.edu
The goal of this paper is to introduce more control over the sampling of the remeshed model. In particular, our method creates rarefied sampling according to a given direction field. Since the parametric region (a unit square) is fixed, that will incur a denser sampling in other regions of the surface. We can therefore adjust surface sampling in a desired way. An example of a controlled stretching of the parameterization away from a corner of the Rockerarm mesh patch is shown in Fig. 1.

This research originated from the desire to have more control over parameterization without introduction of rigid constraints on the parameterization. This can be useful in applications where the regular mesh density needs to be adjusted: instead of specifying the regions where the mesh needs to be denser, we specify the regions where the regular sampling can be sparser in a certain direction.

A possible application of our scheme can produce better surface approximations by optimizing the direction field to sparsify regions with low curvature to pull the sampling of the reconstructed regular meshes towards the areas with higher curvature. An alternative approach to this problem was recently presented by Sander et al. [4].

In the following sections we formulate a variant of Floater's scheme in terms of second difference minimization similar to that in [10]. This allows us to incorporate anisotropic stretching modification in a natural way by adding another term to the minimization functional. Our work is similar to the approach of [11].

\section{Mesh parameterization}

Notation: We consider a triangular manifold mesh $\mathcal{M}=(\mathcal{V}, \mathcal{T})$ with the vertex set $\mathcal{V}$ and the face set $\mathcal{T}$, and a "coordinate" function $\mathbf{x}: \mathcal{V} \rightarrow \mathbf{R}^{3}$. When using a local parameterization on a small neighborhood of the mesh, we shall reserve $\xi=\left(\xi^{1}, \xi^{2}\right)$ for such parameterization. A typical local parameterization of the 


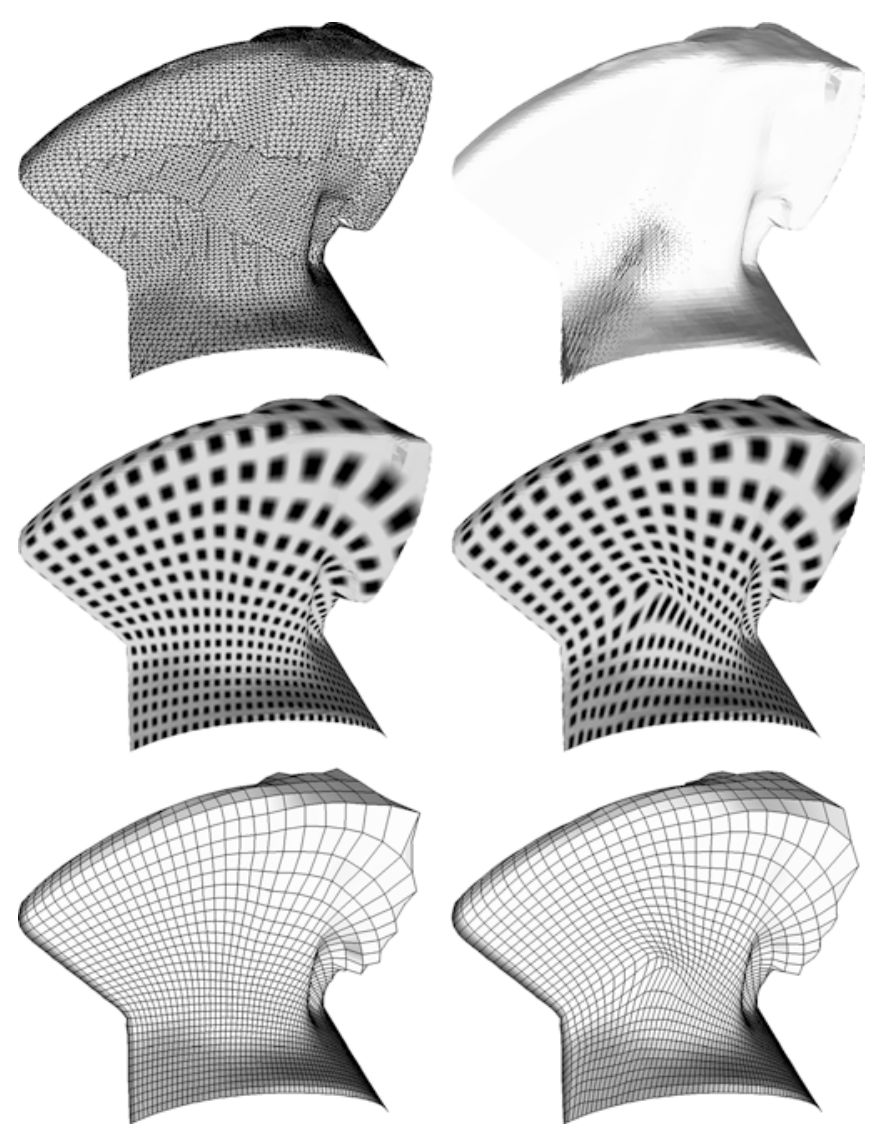

Fig. 1 Floater's original isotropic and our anisotropic parameterizations of a patch of the Rockerarm mesh. Top: original mesh and a direction field. Middle: parameterizations. Bottom: resulting regular remeshes

"umbrella" of faces adjacent to a given vertex can be obtained by flattening such a neighborhood via a "polar map" as described in [9, 12].

Parameterization bijectively maps a mesh region onto a planar region. In remeshing applications a regularly sampled mesh is the goal, and it is therefore typical that the boundary of a mesh patch is mapped onto the boundary of a simple plane region (e.g., a square) in a fixed way. The parameterization scheme is then often specified by per-inner-vertex relations: both linear [1, 9] and nonlinear [7] approaches are employed in practice. When linear equations are used, the bijectivity of the parametric mapping can be ensured by introducing a convexity condition.

Formally, the goal of parameterization is to find a parametric function $U: \mathcal{V} \rightarrow \mathbf{R}^{2}$ such that it maps the boundary vertices of the mesh $\mathcal{M}$ onto the boundary of a simple planar region (we shall use a unit square in this paper), while the inner vertices are mapped inside the square with the condition that the corresponding piecewise linear map based on $U$ and $\mathcal{T}$ is injective (there are no flipped triangles in the parametric region). See [13] for more details. Technicalities aside, a sufficient condition for having a bijection between the planar square and the original mesh is that the parameteric function $U=\left(U^{1}, U^{2}\right)$ satisfies a convex relation at every inner vertex of the mesh, so that for every inner vertex $\bar{v}$ and every vertex $v$ in its one-ring $\omega(\bar{v})$ there exist real weights $\alpha_{\bar{v} v}$ such that

$$
\sum_{v \in \omega(\bar{v})}^{\alpha_{\bar{v} v}>0} \alpha_{\bar{v} v}=1 \quad \text { (positivity) }
$$

and

$\sum_{v \in \omega(\bar{v})} \alpha_{\bar{v} v} U(v)=U(\bar{v})$

Such parameterization is called a convex combination $\operatorname{map}[13]$.

Hence, one approach to parameterization will be to specify a collection of weights satisfying the previously mentioned condition, and then find the parameterization inside the mesh region by solving the resulting linear system. As we shall see later, the affineness condition is easy to ensure by restricting ourselves to a certain class of schemes, while the positivity condition is harder to achieve.

\section{Second differences}

Consider a real function $f: \mathcal{V} \rightarrow \mathbf{R}$ (think of $f=U^{1}$ or $f=U^{2}$ ). Given an assignment of the local neighborhood parameterizations we treat $f$ as samples of a function from $\mathbf{R}^{2}$ to $\mathbf{R}$. More precisely, let $\Omega$ be a neighborhood submesh where the local parameterization $\xi$ is established. The parameterization $\xi$ is thus defined on the vertex set $\mathcal{V}_{\Omega}$ of $\Omega$ (e.g., $\mathcal{V}_{\Omega}=\omega(\bar{v}) \cup \bar{v}$ for some vertex $v$ ).

Given a triple of vertices $v_{1}, v_{2}, v_{3} \in \mathcal{V}_{\Omega}$ we define the first divided difference $D_{\left\{v_{1}, v_{2}, v_{3}\right\}}^{[1]} f$ of $f$ on the $D^{[1]}$ stencil $\left\{v_{1}, v_{2}, v_{3}\right\}$ as the slope of the linear interpolant passing through these three sample values; thus

$D_{\left\{v_{1}, v_{2}, v_{3}\right\}}^{[1]} f=\mathbf{V}^{-1} \Delta f$,

where $\Delta f=\left[f_{2}-f_{1}, f_{3}-f_{1}\right]^{\mathrm{T}}$ and the matrix $\mathbf{V}$ is given as

$\mathbf{V}=\left[\begin{array}{ll}\xi_{2}^{1}-\xi_{1}^{1} & \xi_{2}^{2}-\xi_{1}^{2} \\ \xi_{3}^{1}-\xi_{1}^{1} & \xi_{3}^{2}-\xi_{1}^{2}\end{array}\right]$,

where $\xi_{k}^{i}:=\xi^{i}\left(v_{k}\right)$.

Given two $D^{[1]}$ stencils that share a pair of vertices it is easy to see that the difference between the corresponding first divided differences is always orthogonal to the line connecting the two common points. Thus, a scalar quantity measuring closeness of first derivatives of a sampled function can be introduced as a projection of the previously mentioned difference onto the corresponding normal direction. More precisely, let $\left\{v_{1}, v_{2}, v_{3}\right\}$ and $\left\{v_{2}, v_{3}, v_{4}\right\}$ be the $D^{[1]}$ stencils under consideration. We define the unit vector orthogonal to the edge $\left[\xi\left(v_{2}\right), \xi\left(v_{3}\right)\right]$ as

$\mathbf{n}_{23}=\left[\left(\xi_{3}^{2}-\xi_{2}^{2}\right) /\left\|\xi_{3}-\xi_{2}\right\|,\left(\xi_{2}^{1}-\xi_{3}^{1}\right) /\left\|\xi_{3}-\xi_{2}\right\|\right]$. 
The second difference $\Delta_{\left(v_{1}, v_{2}, v_{3}, v_{4}\right)}^{[2]} f$ is then defined via

$$
\begin{aligned}
\Delta_{\left(v_{1}, v_{2}, v_{3}, v_{4}\right)}^{[2]} f & =\mathbf{n}_{23} \cdot\left(D_{\left\{v_{1}, v_{2}, v_{3}\right\}}^{[1]} f-D_{\left\{v_{2}, v_{3}, v_{4}\right\}}^{[1]} f\right) \\
& =c_{1} f\left(v_{1}\right)+c_{2} f\left(v_{2}\right)+c_{3} f\left(v_{3}\right)+c_{4} f\left(v_{4}\right),
\end{aligned}
$$

where

$$
\begin{array}{lc}
c_{1}=\frac{\left\|\xi\left(v_{2}\right)-\xi\left(v_{3}\right)\right\|}{S_{123}}, & c_{2}=-\frac{\left\|\xi\left(v_{2}\right)-\xi\left(v_{3}\right)\right\| S_{314}}{S_{123} S_{432}}, \\
c_{3}=-\frac{\left\|\xi\left(v_{2}\right)-\xi\left(v_{3}\right)\right\| S_{241}}{S_{123} S_{432}}, & c_{4}=\frac{\left\|\xi\left(v_{2}\right)-\xi\left(v_{3}\right)\right\|}{S_{432}},
\end{array}
$$

and the signed areas $S_{i j k}$ are given as

$$
S_{i j k}:=\operatorname{det}\left[\begin{array}{ccc}
1 & \xi^{1}\left(v_{i}\right) & \xi^{2}\left(v_{i}\right) \\
1 & \xi^{1}\left(v_{j}\right) & \xi^{2}\left(v_{j}\right) \\
1 & \xi^{1}\left(v_{k}\right) & \xi^{2}\left(v_{k}\right)
\end{array}\right] .
$$

Put simply the second difference characterizes the change of slope across an edge shared by two triangles.

In [10] second differences were used in the construction of multiresolution subdivision filters on irregular mesh hierarchies, and the $D^{[1]}$ stencils came from triangles of the mesh. In the following section we show how Floater's shape-preserving scheme can be constructed using second differences.

\subsection{Floater's parameterization scheme}

We follow Floater [9] and consider a one-ring neighborhood of a particular inner vertex of a triangular mesh. Let $\bar{v}$ be the center vertex of the one ring, and $\omega(\bar{v})=\left\{v_{0}, \ldots, v_{n-1}\right\}$ be the set of its neighbors indexed consistently in a counterclockwise order (Fig. 2). Floater considers a vertex $v_{k} \in \omega(\bar{v})$ and finds an index $l=l(k)$ such that the ray $\overrightarrow{\xi\left(v_{k}\right) \xi(\vec{v})}$ intersects the segment $\operatorname{conv}\left\{\xi\left(v_{l}\right), \xi\left(v_{l+1}\right)\right\}$ (all indices in one ring are treated modulo vertex valence $n)$. This ensures that $\xi(\bar{v})$ lies inside the triangle $\operatorname{conv}\left\{\xi\left(v_{l}\right\}, \xi\left(v_{l+1}\right), \xi\left(v_{k}\right)\right\}$. (Fig. 2b). At this point, we look at the second difference

$$
\begin{aligned}
& g_{[2]}\left(\bar{v}, v_{k}\right):=\Delta_{\left(\bar{v}, v_{l(k)}, v_{l(k)+1}, v_{k}\right)}^{[2]} f= \\
& c_{\bar{v}}^{\bar{v}, v_{k}} f(\bar{v})+c_{v_{k}}^{\bar{v}, v_{k}} f\left(v_{k}\right)+c_{v_{l}}^{\bar{v}, v_{k}} f\left(v_{l}\right)+c_{l+1}^{\bar{v}, v_{k}} f\left(v_{l+1}\right),
\end{aligned}
$$

and notice that three coefficients $c_{v_{k}}^{\bar{v}, v_{k}}, c_{v_{l}}^{\bar{v}, v_{k}}, c_{v_{l+1}}^{\bar{v}, v_{k}}$ have the same sign, while $c_{\bar{v}}^{\bar{v}, v_{k}}$ is of the opposite sign. With a bit more precision we claim the following is true:

(a)

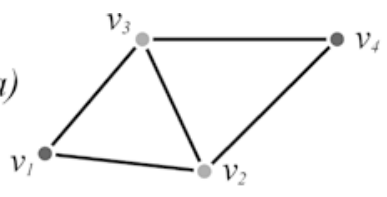

(b)

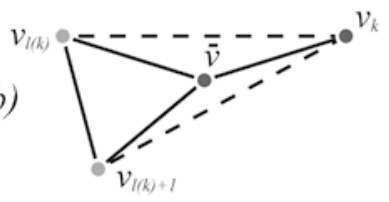

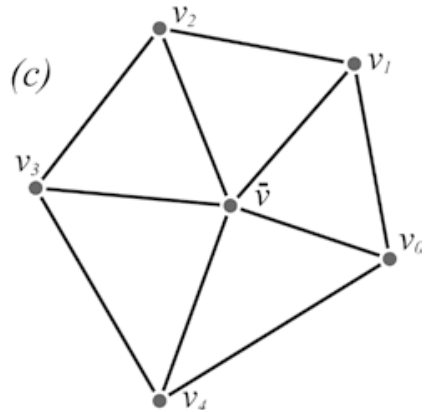

Fig. 2 a A typical $\Delta^{[2]}$ stencil from [10]; b a typical $\Delta^{[2]}$ stencil used in Floater's scheme; $c$ one-ring indexing for Floater's scheme $c_{v_{k}}^{\bar{v}, v_{k}} c_{\bar{v}}^{\bar{v}, v_{k}}<0, \quad c_{v_{l}}^{\bar{v}, v_{k}} c_{\bar{v}}^{\bar{v}, v_{k}} \leqslant 0, \quad c_{v_{l+1}}^{\bar{v}, v_{k}} c_{\bar{v}}^{\bar{v}, v_{k}} \leqslant 0$.

Note: One of the $D^{[1]}$ stencils participating in $g_{[2]}$ is no longer aligned with a triangle from $\mathcal{T}$.

In order to obtain a linear predictor $P_{\bar{v}}$ for the function value at the center vertex $\bar{v}$ given the neighboring values we minimize the following functional which is quadratic in $f(\bar{v})$ (we label it $J^{\text {iso }}$ for being isotropic):

$J_{\bar{v}}^{\text {iso }}(f)=\sum_{k=0}^{n-1}\left[g_{[2]}\left(\bar{v}, v_{k}\right)\right]^{2}$.

The desired value of the function at the center vertex is then given as

$P_{\bar{v}} f=\operatorname{argmin}_{f(\bar{v})} J_{\bar{v}}^{\text {iso }}(f)$.

One can easily check that the corresponding parameterization scheme is identical to the scheme of Floater [9]. Indeed, for the optimal value of $f(\bar{v})$ we have

$$
\begin{aligned}
\sum_{k} c_{\bar{v}}^{\bar{v}, v_{k}}\left[c_{\bar{v}}^{\bar{v}}, v_{k} f(\bar{v})+c_{\bar{v}_{k}}^{\bar{v}, v_{k}} f\left(v_{k}\right) .\right. \\
\left.\quad+c_{v_{l(k)}, v_{k}}^{\bar{v}} f\left(v_{l(k)}\right)+c_{v_{l(k)+1}, v_{k}}^{\bar{v}} f\left(v_{l(k)+1}\right)\right]=0 .
\end{aligned}
$$

Using the fact that the coefficients of the second difference operator always sum up to zero, we obtain the following linear relation

$$
\left(\sum_{s} A_{s}^{\text {iso }}\right) f(\bar{v})=\sum_{s} A_{s}^{\text {iso }} f\left(v_{s}\right)
$$

where the coefficients $A_{s}^{\text {iso }}$ are given by

$A_{s}^{\text {iso }}=-c_{\bar{v}}^{\bar{v}, v_{s}} c_{v_{s}}^{\bar{v}, v_{s}}-\sum_{k: s \in\{l(k), l(k)+1\}} c_{\bar{v}}^{\bar{v}, v_{k}} c_{v_{s}}^{\bar{v}, v_{k}}$.

Note that $A_{s}^{\text {iso }}$ are guaranteed to be positive because of Eq. (1).

Thus, introducing $\alpha_{s}^{\text {iso }}=A_{s}^{\text {iso }} / \sum_{s} A_{s}^{\text {iso }}$, we get the center vertex function value as a convex combination of surrounding values:

$f(\bar{v})=\sum_{s} \alpha_{s}^{\text {iso }} f\left(v_{s}\right)$

\subsection{General formulation}

The Floater parameterization scheme described in the previous section considers a collection of local functionals that characterize certain function properties (such as smoothness) and minimize these quantities in the least-squares sense. In the following section we consider a different (enlarged) set of such local functionals, and it will pay off to derive the result of such a minimization problem in the general case. For a similar discussion, see [11]. 
Let $M(\bar{v})$ be a collection of stencils each of which includes a fixed vertex $\bar{v}$. Each stencil $\tau$ from $M(\bar{v})$ is provided with a set of coefficients defining a linear functional $L_{\tau}$ via $L_{\tau} f:=\sum_{v \in \tau} \lambda_{\tau, v} f(v)$. We assume that all $L_{\tau}$ 's used in our constructions annihilate constants, so

$\sum_{v \in \tau} \lambda_{\tau, v}=0$

We also assume that these stencils cover the one-ring of the vertex $\bar{v}$, i.e.,

$$
\bigcup_{\tau \in M(\bar{v})} \tau=\omega(\bar{v}) \cup\{\bar{v}\} .
$$

In order to find the value of the function at the center vertex, we form $J_{\bar{v}}(f):=\sum_{\tau \in M(\bar{v})}\left(L_{\tau} f\right)^{2}$ and minimize it with respect to the value of the function at the "center " vertex $f(\bar{v})$.

Differentiating $J_{\bar{v}}$ with respect to $f(\bar{v})$, we can easily see that the resulting scheme should have the following form:

$\sum_{\tau \in M(\bar{v})}\left(\lambda_{\tau, \bar{v}} f(\bar{v})+\sum_{v \in \tau \backslash\{\bar{v}\}} \lambda_{\tau, v} f(v)\right) \lambda_{\tau, \bar{v}}=0$.

Rearranging the terms and using Eq. (2), we obtain

$$
\left(\sum_{v \in \omega(\bar{v})} A_{v}\right) f(\bar{v})=\sum_{v \in \omega(\bar{v})} A_{v} f(v),
$$

where

$A_{v}:=-\sum_{\tau \in M(\bar{v}): \tau v} \lambda_{\tau, v} \lambda_{\tau, \bar{v}}$.

It is immediately clear that the affineness condition always holds for the schemes constructed in this way. On the other hand a sufficient condition for the scheme to have positive coefficients is that every contributing stencil has its coefficients for noncenter vertices of the opposite sign to its coefficient for the center vertex. This condition holds for Floater's scheme construction in the previous section.

Example: A simple example of a parameterization scheme can be obtained by considering the set of functionals

$$
\left\{\Delta_{i} f:=f\left(v_{i}\right)-f(\bar{v}): i=0, \ldots, n-1\right\} .
$$

Then the corresponding stencils are $\tau_{i}:=\left\{\bar{v}, v_{i}\right\}$ and the coefficients $\lambda$ are given as

$\lambda_{\tau_{i}, \bar{v}}=-1, \quad \lambda_{\tau_{i}, v_{i}}=1$.

Hence, $A_{v}=1$ and the resulting parameterization scheme is a simple regular umbrella:

$n f(\bar{v})=\sum_{i=0}^{n-1} f\left(v_{i}\right)$.

\section{Anisotropic modification}

In this section we introduce a modification to the original Floater scheme that can produce anisotropic parameterization that is stretched in a direction given by a direction field on the surface. We shall use a direction field specified as a vector on each face of the mesh. The direction fields can be specified by the user or can be created automatically. In our implementation the user specifies a desired rough direction field, which is then smoothed using a procedure similar to the approach presented in [14].

\subsection{A simple scheme}

The direction field is given by a vector represented in the local coordinate system of each triangle of the mesh. It turns out that the fact that the vector field is directed does not matter for the derivation of the anisotropic scheme. Formally, after the local neighborhood parameterization $\xi$ is fixed, we have an assignment of direction vectors to triangles $h: \mathcal{T} \rightarrow \mathbf{R}$ that samples a vector field $\mathbf{H}=H^{\prime} \partial / \partial \xi^{\prime}$. The anisotropic scheme will stretch both parametric functions $U^{j}, j=1,2$, in the direction of $\mathbf{H}$. To achieve that effect we add an extra term representing $\left(\mathbf{H} U^{j}\right)^{2}$ into the minimization of $J\left(U^{j}\right)$. As a result the derivative in the direction of $\mathbf{H}$ will get smaller, introducing a stretch along the given direction field. Note that the "negated" vector field $-\mathbf{H}$ will result in the same stretch, and thus the directionality of $\mathbf{H}$ does not matter at the parameterization stage (it does matter in the vector-smoothing step that produces $\mathbf{H}$, see [14]).

The discrete implementation of this approach is straightforward: we replace the partial derivatives by the first divided difference operator and add the sum of the squares of the resulting quantities to the isotropic functional. This yields an anisotropic functional $J^{\text {anis }}$

$J_{\bar{v}, \beta}^{\text {anis }}[f(\bar{v})]=\sum_{k=0}^{n-1}\left\{\left[g_{[2]}\left(\bar{v}, v_{k}\right)\right]^{2}+\beta\left[f_{[1], h}\left(\bar{v}, v_{k}, v_{k+1}\right)\right]^{2}\right\}$

where the first part of the sum is copied from the isotropic case and the quantities in the second term are defined for a general vertex triple $t=\left\{v_{0}, v_{1}, v_{2}\right\}$ and an associated direction vector $\mathbf{h}_{t}$ via

$$
\begin{aligned}
f_{[1], h}\left(v_{0}, v_{1}, v_{2}\right) & =h\left(\left\{v_{0}, v_{1}, v_{2}\right\}\right) \cdot D_{\left\{v_{0}, v_{1}, v_{2}\right\}}^{[1]} f \\
& =d_{0} f\left(v_{0}\right)+d_{1} f\left(v_{1}\right)+d_{2} f\left(v_{2}\right),
\end{aligned}
$$

where

$$
\begin{aligned}
& d_{0}=\frac{1}{S_{012}}\left\{\left[\xi^{2}\left(v_{1}\right)-\xi^{2}\left(v_{2}\right)\right] h_{t}^{1}+\left[\xi^{1}\left(v_{2}\right)-\xi^{1}\left(v_{1}\right)\right] h_{t}^{2}\right\}, \\
& d_{1}=\frac{1}{S_{012}}\left\{\left[\xi^{2}\left(v_{2}\right)-\xi^{2}\left(v_{0}\right)\right] h_{t}^{1}+\left[\xi^{1}\left(v_{0}\right)-\xi^{1}\left(v_{2}\right)\right] h_{t}^{2}\right\}, \\
& d_{2}=\frac{1}{S_{012}}\left\{\left[\xi^{2}\left(v_{0}\right)-\xi^{2}\left(v_{1}\right)\right] h_{t}^{1}+\left[\xi^{1}\left(v_{1}\right)-\xi^{1}\left(v_{0}\right)\right] h_{t}^{2}\right\} .
\end{aligned}
$$

We can then repeat the derivation of the previous section to obtain 

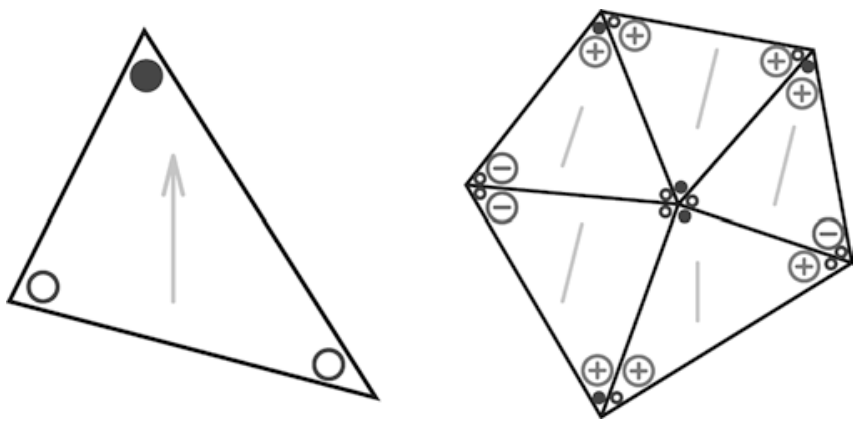

Fig. 3 Left: the signs of the coefficients for the $f_{[1], h}$ operator. Right: the signs of the contributions to the $A_{s}^{\text {stretch }}$ coefficients for the given distribution of direction vectors

$$
\left(\sum_{s} A_{s}^{\text {anis }}\right) f(\bar{v})=\sum_{s} A_{s}^{\text {anis }} f\left(v_{s}\right),
$$

with $A_{s}^{\text {anis }}$ derived from Eq. (3).

So far we took no special care to ensure the positivity of the coefficients $A_{s}^{\text {anis}}$; hence, there is no guarantee that the resulting linear system is well-defined. It is clear, however, that given a bounded direction field, $J_{\bar{v}, \beta}^{\text {anis }}$ will produce a convex combination map scheme for small values of $\beta$. As we explain in the next section, clamping the value of $\beta$ to the maximum allowed by positivity

Fig. 4 Clamped anisotropic (left) and nonclamped anisotropic schemes for the Mannequin model. The values of $\beta$ are 100 (top), 400 (middle), and 1,600 (bottom). It is easy to see distortions and nonconvexity for the scheme without positivity correction
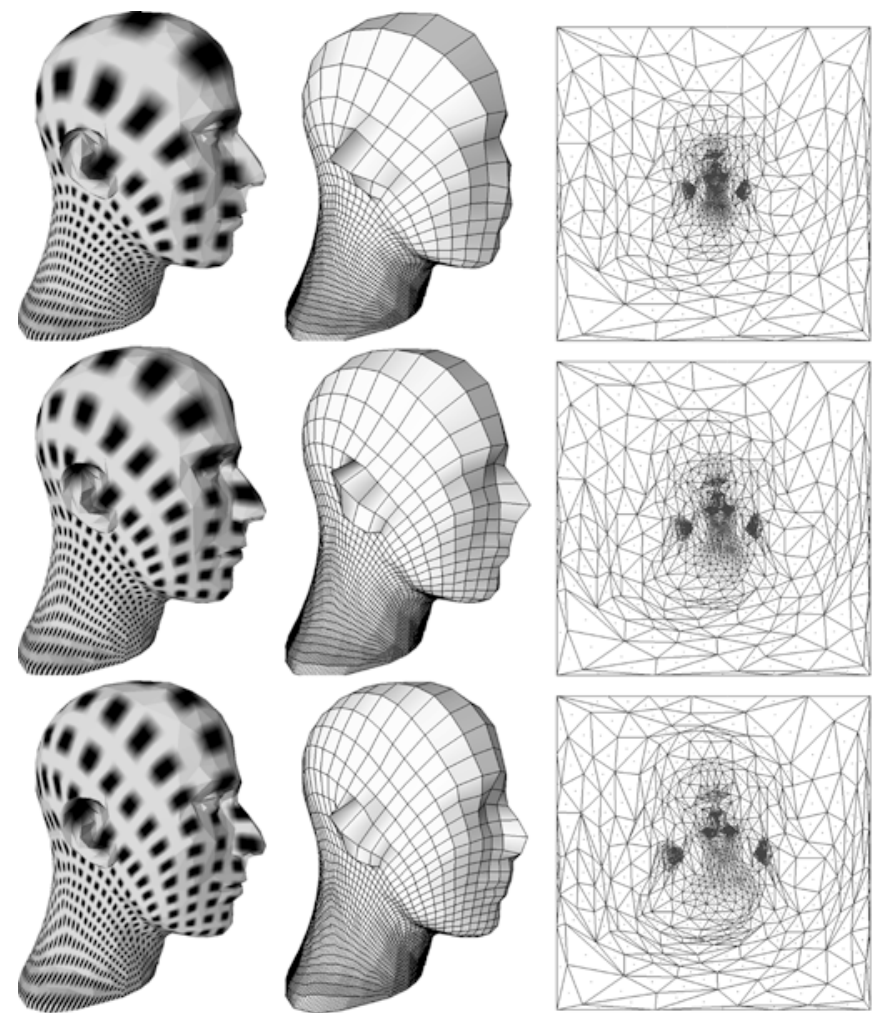

condition turns out to produce a very practical anisotropic parameterization scheme.

\subsection{Positivity}

In this section we discuss possible approaches to making the scheme monotone. First, we look at the stencil for directed derivatives. The main issue is the signs of the coefficients. Let $\tau=\left\{\bar{v}, v_{1}, v_{2}\right\}$ be a stencil of three vertices. Then it will contribute monotonely to the scheme centered at $\bar{v}$ if the direction $h$ splits the angle $\xi\left(v_{1}\right) \widehat{\xi(\bar{v})} \xi\left(v_{2}\right)$. Formally, a vector $\mathbf{h}=\left(h^{1}, h^{2}\right)$ splits an angle $\widehat{\xi \eta \zeta}$ if $h^{\perp} \cdot(\xi-\eta)$ and $h^{\perp} \cdot(\zeta-\eta)$ are of opposite signs [we use $h^{\perp}=\left(h^{2},-h^{1}\right)$ ]. Note that within a triangle, only one of the angles is split by a given direction vector (this angle is denoted with a filled circle in Fig. 3). The coefficient of $f_{[1], h}$ for the split corner is opposite in sign to the other two coefficients (this can be seen visually by varying the values of the function one by one, since $f_{[1], h}$ gives the slope of the function in the given direction).

One conservative way to ensure the positivity of the scheme is to make sure that a triangle's anisotropic term only contributes to $J_{\bar{v}, \beta}^{\text {anis }}$ for the vertex $\bar{v}$ whose corner is split by the direction given on that triangle. This is easy to implement; however, our experiments show that the resulting scheme does not produce sufficient stretching even for large values of $\beta$.

We therefore adapt a less conservative and somewhat simpler approach that is mentioned in the previous section. Namely, for every inner vertex $\bar{v}$ we clamp the value of $\beta$ to be less than the precomputed value $\beta_{\max }$

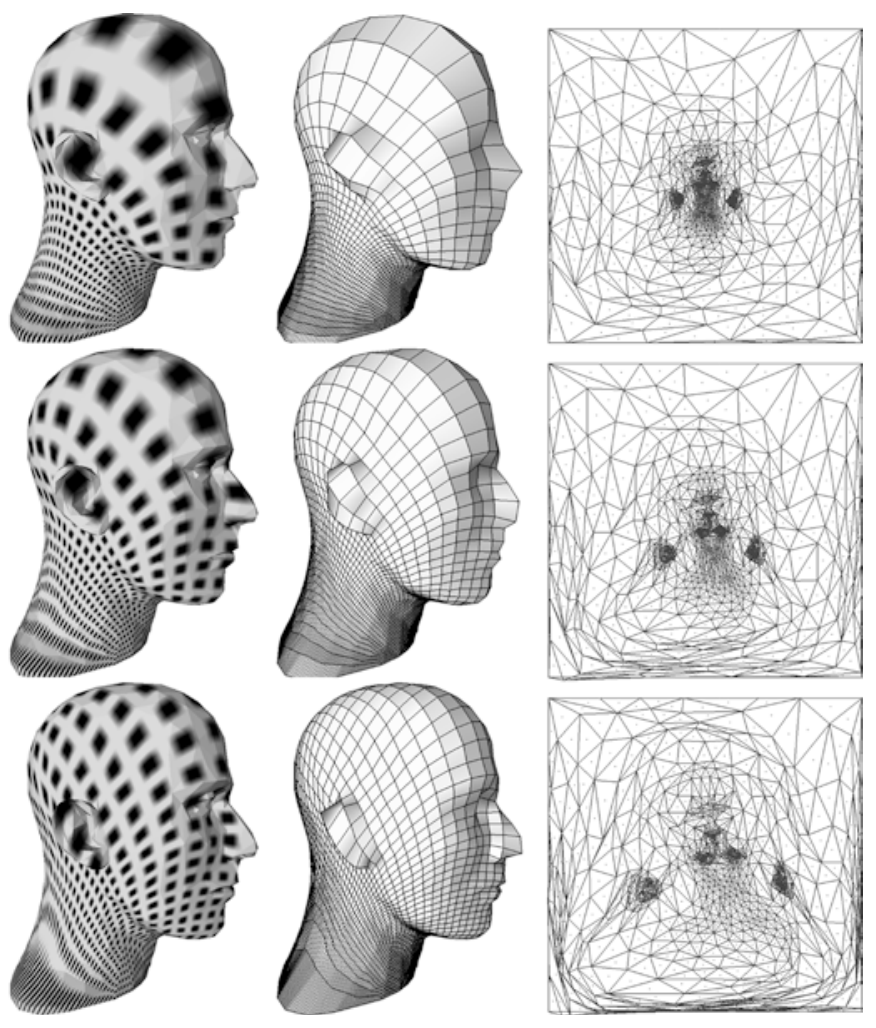


that ensures the positivity of the scheme. We can find such $\beta_{\max }$ by noting that the coefficients $A_{s}^{\text {anis }}$ are combinations of the isotropic Floater coefficients and the "stretching" coefficients (we can do so by splitting the stencils of Eq. 3 into two groups). Hence, we obtain

$A_{s}^{\text {anis }}=A_{s}^{\text {iso }}+\beta A_{s}^{\text {stretch }}$,

where some of $A_{s}^{\text {stretch }}$ can be negative. We then find

$\beta_{\max }(\bar{v})=\min _{s} \frac{A_{s}^{\text {iso }}}{-\min \left(0, A_{s}^{\text {stretch }}\right)}$,

(note that we get a value of infinity if all the coefficients $A_{s}^{\text {stretch }}$ are positive, with the meaning that there is no restriction on $\beta(\bar{v})$.

Then the new "clamped anisotropic" scheme is obtained from

$J_{\bar{v}, \beta}^{\text {ca }}(f):=J_{\bar{v}}^{\text {iso }}(f)+\min \left[\beta, \beta_{\max }(\bar{v})\right] J_{\bar{v}}^{\text {stretch }}(f)$,

where $J_{\bar{v}}^{\text {stretch }}(f)=\sum_{k=0}^{n-1} f_{[1], h}\left(\bar{v}, v_{k}, v_{k+1}\right)[]^{2}$.

\subsection{Results}

We demonstrate the performance of the schemes described in previous sections for a number of examples. The parameterizations produced with our scheme are visualized in a number of ways: as the mapping of a regular texture using the parametric functions produced, as the resampling of the original surface, and as the picture of the original mesh in the parametric $\left(U^{1}, U^{2}\right)$ plane.

The performance of the clamped scheme that guarantees the convexity condition is compared in Fig. 4 with that of the nonclamped scheme of Sect. 3.1. It is clear that for the same value of parameter $\beta$ the clamped scheme will have less stretching than the nonclamped one. Thus, a similar parameterization shift (and change in regular sampling frequency) is achieved at different values of $\beta$ for the two schemes considered. It is also clear that the nonclamped scheme results in some noninjective parameterizations (note the triangles mapped outside of the unit square in the bottom row of the right column).

The effect of the anisotropic stretching in the parametric plane is illustrated in Fig. 5. The parametric

Fig. 5 Square anisotropic parameterization for $\beta=0.800,6,300$, and 25,600
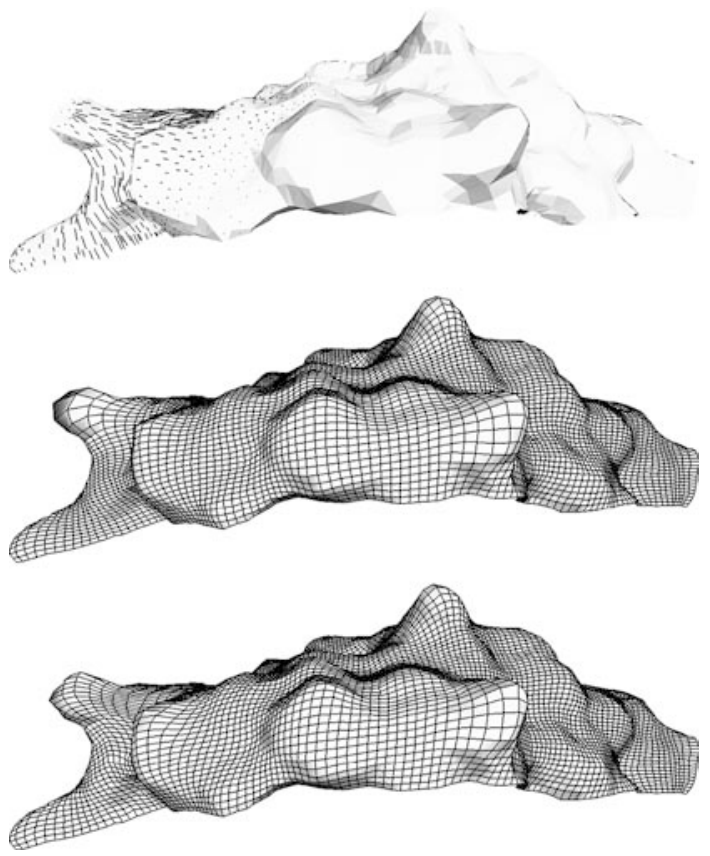

Fig. 6 Focusing the sampling onto a high-curvature geometric feature for a Molecule mesh patch. Top: direction field on the original surface. Middle: isotropic remesh. Bottom: anisotropic remesh using the given direction field for $\beta=400$

function becomes "flatter" in the given directions, which results in bringing the points closer in the parametric domain. Thus, we see a close packing of squeezed triangles in a certain region of the triangulation. The role of the convexity condition is therefore to keep the triangles from flipping and riding on top of other triangles.

Figure 6 uses the direction field to focus the sampling onto a high-curvature feature of the Molecule model. The direction field vectors point away from the feature and are zero on the feature itself. This results in allowing more samples to be placed onto the feature, resulting in a better approximation.

A simple way to introduce a wiggle pattern for the parameterization lines is shown in Fig. 7. Two stretching regions push the samples of the remeshed model away from them.

Performance note: The schemes introduced produce a linear system of equations that is solved with a biconjugate gradient method. Since the mesh sizes are small (maximum of tens of thousands of vertices) all the computations take a matter of seconds.
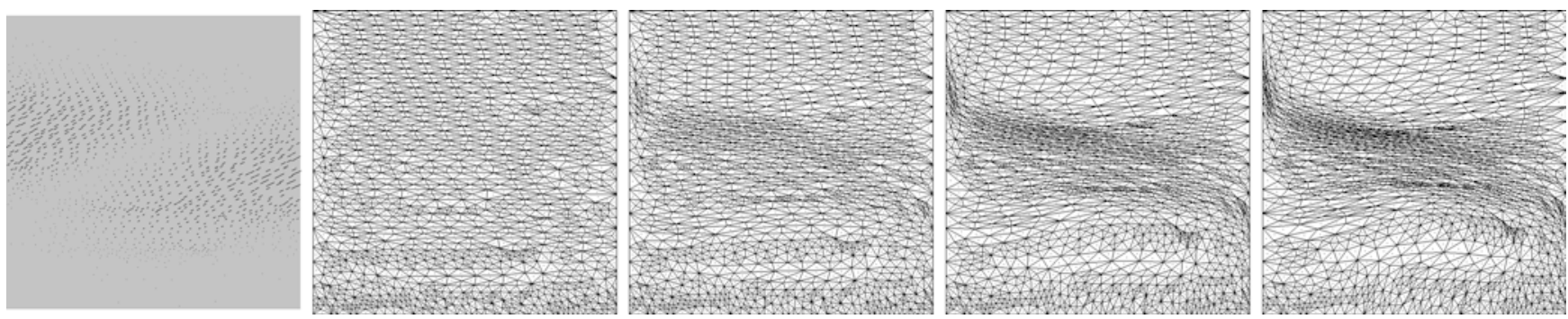


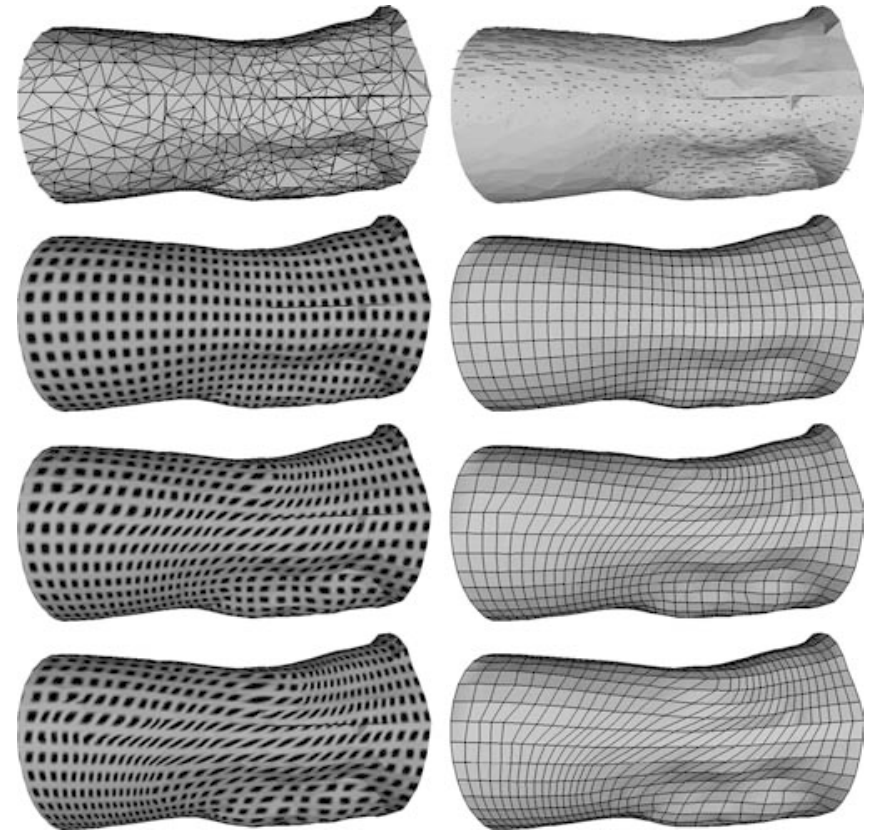

Fig. 7 Clamped anisotropic scheme for the Knee model: the first row shows the original mesh and the direction field, the second row shows the parameterization and regular remesh for $\beta=0$, the third row shows the parameterization and regular remesh for $\beta=1,000$, and the fourth row shows the parameterization and regular remesh for $\beta=10,000$

\section{Conclusions}

We have introduced a simple anisotropic modification of Floater's shape-preserving parameterization scheme that allows flattening of parametric mapping along a given direction field. Future work includes the development of algorithms for constructing direction fields that allow better approximation of geometric features as well as construction of unconditionally positive schemes. Another interesting question is whether one can improve the scheme performance via selective local mesh refinement. Currently there are no estimates for the shift in the parameterization in relation to the changes in the anisotropic parameter $\beta$. Finding such a relation is important for applying our scheme to automated parameterization adjustment. Extension of our approach to $3 \mathrm{D}$ parameterization is also a possibility.

Acknowledgements This work was supported in part by NSF (CCR-0133554). The models are courtesy of Cyberware, the University of Washington, and the Scripps Institute

\section{References}

1. Hoppe H, DeRose T, Duchamp T, Halstead M, Jin H, McDonald J, Schweitzer J, Stuetzle W (1994) Piecewise smooth surface reconstruction. Proceedings of SIGGRAPH, pp 295 302

2. Krishnamurthy V, Levoy M (1996) Fitting smooth surfaces to dense polygon meshes. Proceedings of SIGGRAPH, pp 313324

3. Praun E, Finkelstein A, Hoppe H (2000) Lapped textures. Proceedings of SIGGRAPH, pp 465-470

4. Sander PV, Snyder J, Gortler SJ, Hoppe H (2001) Texture mapping progressive meshes. Proceedings of SIGGRAPH, pp 409-416

5. Guskov I, Vidimče K, Sweldens W, Schröder P (2000) Normal meshes. Proceedings of SIGGRAPH, pp 95-102

6. Alliez P, Meyer M, Desbrun M (2002) Interactive geometry remeshing. Proceedings of SIGGRAPH

7. A.Sheffer, de Sturler E (2000) Surface parameterization for meshing by triangulation flattening. Proceedings of the 9th international meshing roundtable, pp 161-172

8. Lévy B, Mallet J (1998) Non-distorted texture mapping for sheared triangulated meshes.Proceedings of SIGGRAPH, pp 343-352

9. Floater MS (1997) Parameterization and smooth approximation of surface triangulations. Comput -Aided Geomet Des $14: 231-250$

10. Guskov I, Sweldens W, Schröder P (1999) Multiresolution signal processing for meshes. Proceedings of SIGGRAPH, pp 325-334

11. Lévy B (201) Constrained texture mapping for polygonal meshes. Proceedings of SIGGRAPH, pp 417-424

12. Lee AWF, Sweldens W, Schröder P, Cowsar L, Dobkin D (1998) MAPS: multiresolution adaptive parameterization of surfaces. Proceedings of SIGGRAPH, pp 95-104,

13. Floater MS (2001) Convex combination maps. Algorithms for Approximation IV. Huddersfield

14. Hertzmann A, Zorin D (2000) Illustrating smooth surfaces. Proceedings of SIGGRAPH, pp 517-526 